$>$ Les podosomes sont des microdomaines membranaires riches en actine, en interaction directe avec la matrice extracellulaire. Des câbles d'acto-myosine les assemblent en réseau pour former une superstructure cellulaire aux fonctions versatiles. Extensivement décrits in vitro, les podosomes se dessinent comme des acteurs majeurs de processus physiologiques spécifiques. Les détails de leur intervention in vivo restent à préciser. Le microenvironnement ayant un effet prépondérant dans l'acquisition de leurs caractéristiques morphologiques et fonctionnelles, leur rôle ne peut être abordé que dans un contexte cellulaire particulier. Nous nous focaliserons ici sur trois processus impliquant ces structures et discuterons les propriétés des podosomes exploitées dans ces situations. <

Les cellules communiquent en permanence avec leur environnement. Ces interactions sont de nature multiple et impliquent des facteurs solubles, matriciels et mécaniques. Les cellules sondent leur microenvironnement grâce à des filopodes; elles adhèrent aux composants matriciels par le biais d'adhérences focales ou fibrillaires, et déploient des lamellipodes pour migrer. Les podosomes sont des structures indépendantes, formés par un nombre restreint de types cellulaires et/ou dans certaines circonstances. Ils se caractérisent par leurs propriétés protéolytiques vis-à-vis des protéines matricielles et sont, de ce fait, souvent décrits comme des structures permettant l'invasion cellulaire ${ }^{1}$ [1].

La principale composante du microenvironnement est la matrice extracellulaire, un milieu complexe qui se renouvelle en permanence [2]. La matrice interstitielle, majoritaire, est constituée pour une large part de collagène-I fibrillaire, d'élastine et, à un degré moindre, de glycoprotéines, comme la fibronectine et la laminine, leurs proportions variant selon les tissus. La lame

${ }^{1}$ Les invadopodes, des structures apparentées formées par les cellules tumorales, ne seront pas abordées dans cette revue.

\section{Variations sur le thème des podosomes, une affaire de contexte} Florian Alonso ${ }^{1}$, Pirjo Spuul ${ }^{2}$, IJsbrand Kramer ${ }^{1}$,
Elisabeth Génot ${ }^{1}$

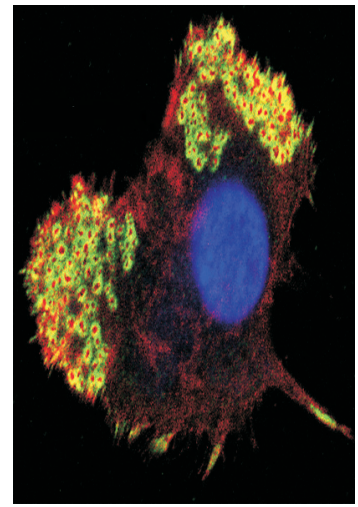

${ }^{1}$ Centre de recherche cardiothoracique de Bordeaux (Inserm U1045), Université de Bordeaux, Bordeaux F-33076 Cedex, France. ${ }^{2}$ Department of chemistry and biotechnology, division of gene technology, Tallinn University of Technology, 12618 Tallinn, Estonie.

elisabeth.genot@inserm.fr

basale représente une matrice d'une autre nature. Composée principalement de laminine et de collagène-IV, elle forme une sorte de treillis compact et résistant qui enveloppe et compartimente les organes tout en délivrant des signaux qui participent à l'homéostasie des tissus.

Structuralement, les podosomes se présentent comme des microdomaines membranaires à base d'actine en contact direct avec les éléments matriciels. D'abord caractérisés comme des structures individuelles, les podosomes sont en réalité les éléments de base d'un réseau, interconnectés par des câbles d'actine et de myosine, l'ensemble associant cytosquelette d'actine et membrane plasmique [3-5]. En tant que domaines membranaires, les podosomes sont des sites privilégiés d'interactions avec l'environnement péricellulaire qui régule leur formation, durée de vie, distribution subcellulaire et architecture. En tant que domaines du cytosquelette, les podosomes dérivent leurs caractéristiques morphologiques et fonctionnelles de la dynamique de polymérisation de l'actine et adoptent un comportement collectif et dynamique $[5,6]$.

Les podosomes sont particulièrement abondants dans les cellules de la lignée myélo-monocytaire comme les macrophages [7], les cellules dendritiques immatures [8] et les ostéoclastes [9], ou encore dans les mégacaryocytes [10]. Dans ces cellules, les podosomes constituent la majeure partie du cytosquelette d'actine et ceci de façon constitutive. Ils peuvent également être formés dans certaines cellules non hématopoïétiques. Ils sont alors inductibles, arrangés en superstructures de forme annulaire (rosettes) et apparaissent en réponse à des signaux bien spécifiques qui dépendent du type de cellules considéré. Leur description in vitro, en milieu bidimensionnel, sous atmosphère 
hyperoxique et en l'absence d'une matrice complexe que sont le milieu interstitiel ou la lame basale, a révélé les multiples potentialités de ces structures. L'objectif est maintenant de préciser leurs présentations et leurs fonctions in vivo.

Du fait du rôle prépondérant du microenvironnement dans l'acquisition de leurs caractéristiques, la fonction des podosomes ne peut être abordée qu'en rapport avec le contexte cellulaire considéré. Nous résumerons dans cette synthèse les dernières avancées dans le domaine, notamment la caractérisation des éléments qui ont permis de mieux cerner l'organisation et la dynamique des podosomes, et leur mise en œuvre dans le cadre des diverses fonctions qui leur sont associées $^{2}$. Nous illustrerons ensuite trois processus physiologiques dans lesquels les podosomes décrits in vitro pourraient intervenir in vivo.

\section{Le podosome, unité de base d'un organite associant cytosquelette et membrane plasmique}

Typiquement, un podosome est un domaine membranaire ponctiforme de 0,5 à $1 \mu \mathrm{m}$ de diamètre dont l'actine, au cœur de la structure, est le composant majoritaire (Figure I A-D) [1]. C'est une structure dynamique, siège d'une activité de polymérisation/dépolymérisation continuelle de l'actine. La polymérisation a lieu au niveau de la membrane plasmique basale de la cellule et, sur un support rigide, la structure ainsi formée croît perpendiculairement au support sur une hauteur de $0,6 \mu \mathrm{m}$ environ (Figure $1 C, D$ ). La cortactine, qui est plus particulièrement impliquée dans la polymérisation de l'actine des podosomes, est souvent utilisée pour les détecter. Tks5 (tyrosine kinase substrate with 5 SH3 domains), une protéine adaptatrice et substrat de Src, joue un rôle essentiel dans la construction des podosomes; elle est également, comme la cortactine, un élément de reconnaissance de ces structures. La métalloprotéase transmembranaire MTl-MMP (ou MMP [matrix metalloproteinase]-14) est un élément clé du podosome. Elle y est délivrée par le réseau de microtubules et est exposée à la membrane plasmique en contact avec la matrice extracellulaire sur laquelle elle exerce son action protéolytique [11]. Du côté cytoplasmique, l'enzyme est associée à l'actine, probablement à différents réseaux, et semble servir de point d'ancrage au cytosquelette [12]. Autour du cœur d'actine, on trouve des protéines décrites dans les adhérences focales, dont la paxilline, la vinculine et la taline, qui connectent le cytosquelette à la membrane plasmique pour assurer l'amarrage de la structure à la matrice via des intégrines (Figure 1C, D) $[1,13]$. La microscopie à haute résolution a révélé que cet anneau, d'apparence uniforme en microscopie de fluorescence classique, est en réalité discontinu, formant un polygone de complexes discrets de protéines d'adhérence autour du cœur $[5,14,15]$. En raison de cette organisation, les podosomes apparaissent comme des structures bipartites lorsque ces composants sont détectés dans un même plan en immunofluorescence (Figure IA). La caractérisation des podosomes dans le plan orthogonal est plus récente. Elle a révélé l'existence

${ }^{2}$ Les aspects concernant la signalisation ne seront pas traités ici. d'un troisième élément qui chapeaute la structure, la coiffe, contenant notamment les formines FMNLl (formin-like 1) [16] et INF2 (inverted formin, FH2 and WH2 domain containing) [17], la supervilline [18] et LSPl (lymphocyte specific protein 1) [19]. Cette structure pourrait servir de plaque tournante pour les vésicules entrantes. Elle pourrait aussi moduler la croissance des podosomes ou encore canaliser les forces contractiles qui leur sont associées [18, 19] (Figure 1C, D).

L'analyse fine des réseaux d'actine a permis de mieux comprendre l'architecture de l'organite et a révélé la mécanique de leurs propriétés sensorielles. Au niveau du cœur, le complexe Arp (actin-related protein) 2/3 est la signature d'un réseau d'actine ramifié [20]; il est entouré d'une enveloppe de filaments non ramifiés, antiparallèles et contractiles, qui rayonnent depuis le sommet du podosome jusqu'à la membrane plasmique ventrale $[4,9,21]$ (Figure 1D). L'utilisation de la microscopie de force atomique a permis de révéler l'oscillation des structures, animées par des échanges de forces [3, 22] : leur cœur croît par polymérisation de l'actine ; les câbles contractiles, amarrés à la matrice au niveau de l'anneau, se tendent et induisent le recrutement de la vinculine à ces sites, et les forces ainsi emmagasinées sont restituées en forces de protrusion au niveau du cœur [5]. La contraction opérée par la myosine-II orchestre cette dynamique $[3,5,22]$. En fonction de la rigidité de la matrice, l'amplitude des forces conduit à différents niveaux de recrutement des composants sensibles à la tension [23], traduisant ainsi la propriété physique du substrat en signaux biochimiques sur les podosomes ; ceux-ci peuvent ensuite être transmis dans la cellule. L'anneau de podosomes est aussi animé par des oscillations, consécutives des mouvements du cœur $[5,6]$. Un autre jeu de câbles d'actine, également non ramifiés et contractiles $[5,18]$, connecte les podosomes les uns aux autres. Ils assurent une distribution régulière $[4,18,24]$ et un comportement collectif et synchrone à plus grande échelle [6] (Figure 1B, D). Les podosomes sont ainsi dotés d'une activité mécanosensorielle, orchestrée par les forces générées en différents points de la structure, qui se combinent ou, plus probablement, alternent avec leur activité protéolytique vis-à-vis de la matrice (Figure I C, D).

\section{Régulation extracellulaire ou intracellulaire de la formation des podosomes}

\section{- Les signaux perçus au niveau de la membrane plas- mique}

Dans les conditions classiques de culture in vitro, sur une surface lisse et rigide, les podosomes se montrent 


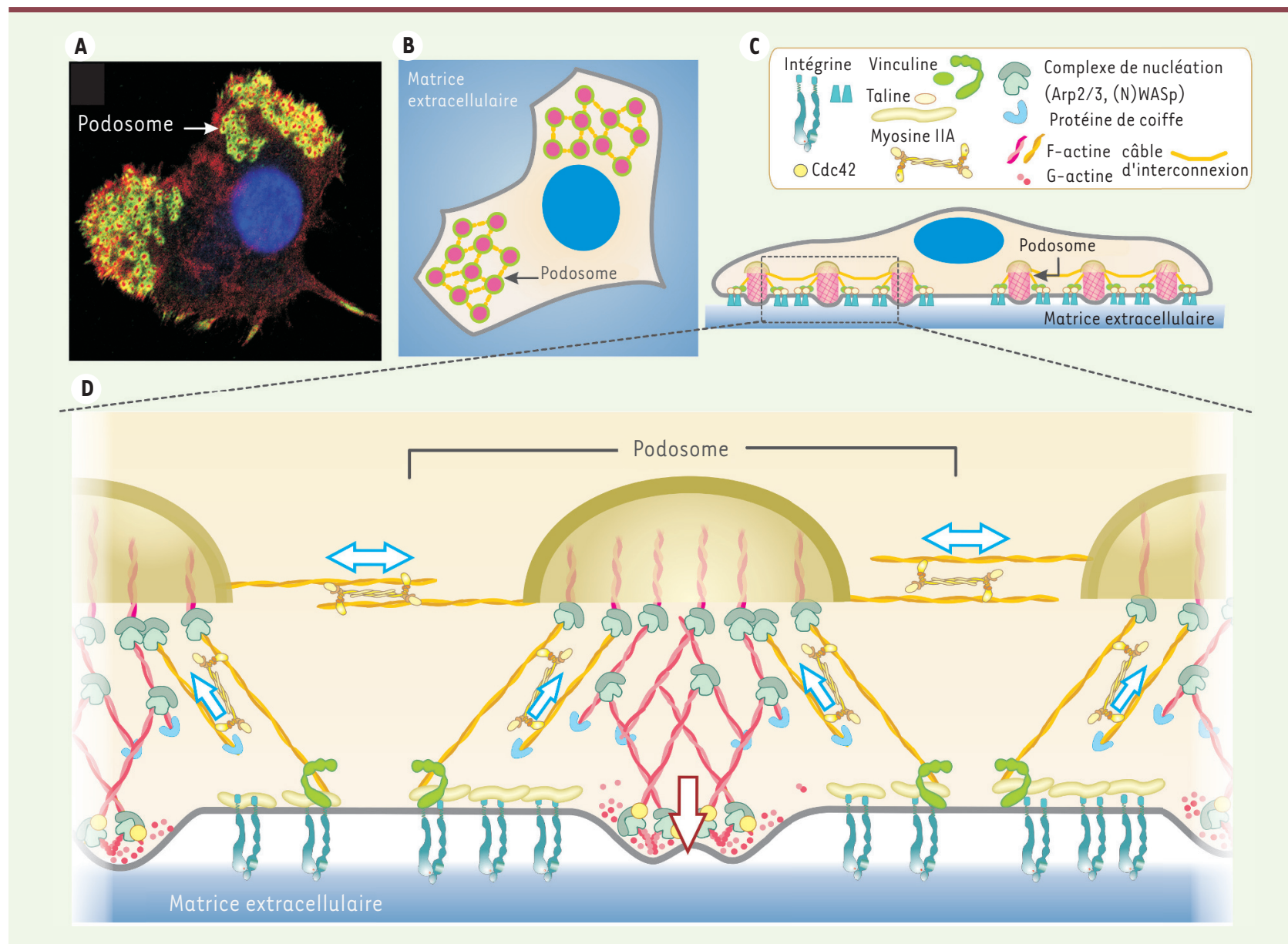

Figure 1. Structure et organisation des podosomes dans une cellule dendritique immature. A. Le marquage immunofluorescent de l'actine (rouge) et de la vinculine (vert), visualisés dans le plan basal, révèle le cœur et l'anneau des podosomes et leur arrangement en groupe. B. Représentation schématique de (A). C. Schéma de la cellule avec ses podosomes en vue sagittale, montrant deux autres éléments récemment découverts: les câbles d'actomyosine qui interconnectent les podosomes et la coiffe de la structure. $D$. Détail d'un podosome : le cœur, siège de la polymérisation de l'actine, l'anneau constitué notamment de protéines permettant l'adhérence à la matrice, la coiffe où terminent les faisceaux de filaments d'actine et les câbles d'actomyosine qui assemblent les podosomes individuels en réseau. Les podosomes sont dotés d'une activité mécanosensorielle orchestrée par les forces générées en plusieurs points (flèches) qui se combinent ou alternent avec leur activité protéolytique vis-à-vis de la matrice (non décrit dans la figure).

sous leur forme caractéristique (Figure 1). Dans le cas des cellules myélomonocytaires, le contact avec le substrat déclenche leur assemblage pour permettre l'adhérence de la cellule. Leur induction reste toutefois fonction de la nature de la matrice extracellulaire et donc de l'engagement d'intégrines spécifiques avec leur signalisation propre (par exemple, l'intégrine $\beta 2$ sur le fibrinogène pour les macrophages [22] et l'intégrine $\beta 3$ sur l'ostéopontine pour les ostéoclastes [25]). Dans le cas des cellules pour lesquelles les podosomes sont inductibles, les signaux matriciels jouent un rôle prépondérant [26-28]: une matrice de fibrinogène est ainsi plus propice à la formation de podosomes par les macrophages [22], alors que ce sera le collagèneIV pour l'induction des podosomes par le VEGF (vascular endothelial growth factor) dans les cellules endothéliales [26]. La matrice module également la durée de vie des podosomes : ceux formés par les mégacaryocytes sur le collagène-I vivent trois fois plus longtemps que ceux formés sur le fibrinogène [10]. En revanche, dans un espace confiné, c'est la fibronectine, et non le collagène-l, qui est permissive à la formation des structures par les cellules endothéliales [28]. La géométrie du substrat dicte la localisation et l'arrangement des podosomes. Sur une surface en relief, les cellules dendritiques accumulent des podosomes le long des crêtes et ceux-ci sont plus stables que ceux qui se sont formés dans les zones non accidentées [29]. En fait, les podosomes s'alignent [10], voire changent de morphologie et de composition [27] pour suivre, par exemple, les méandres des fibres de collagène-I sur lesquelles les cellules ont été ensemencées [10]. Sur une matrice de faible rigidité, les podosomes peinent à se former. Ils s'enfoncent dans la matrice extracellulaire [30]. Inver- 


\begin{tabular}{|c|c|c|c|c|}
\hline Nature du signal & Médiateur & Podosomes & Lit vasculaire & Références \\
\hline \multirow{3}{*}{ Cytokines } & VEGF-A & $\uparrow$ & $\begin{array}{l}\text { Capillaire } \\
\text { Veine }\end{array}$ & {$[26,36,50,51,52]$} \\
\hline & TGF- $\beta$ & $\uparrow$ & Aorte & [56] \\
\hline & TNF- $\alpha$ & $\uparrow$ & Veine & [51] \\
\hline \multirow{3}{*}{ Matrice extracellulaire } & $\begin{array}{l}\text { Collagène-IV } \\
\text { Laminine } 8 / 10\end{array}$ & $\uparrow$ & Capillaire & [26] \\
\hline & $\begin{array}{l}\text { Fibronectine, } \\
\text { Collagène-I }\end{array}$ & $\downarrow$ & Capillaire & [26] \\
\hline & Collagène-I (fibres) & $\uparrow$ & Aorte, capillaire & [27] \\
\hline \multirow{2}{*}{$\begin{array}{l}\text { Communication } \\
\text { intercellulaire }\end{array}$} & Voie Notch & $\downarrow$ & Capillaire & {$[36]$} \\
\hline & Exosomes & $\uparrow$ & Capillaire & [38] \\
\hline Métabolisme & $\begin{array}{l}\text { Espèce réactives de } \\
\text { l'oxygène, nutriments }\end{array}$ & $\uparrow$ & Capillaire & [38] \\
\hline Micro ARN & miR-155 & $\uparrow$ & Aorte & [34] \\
\hline \multirow{2}{*}{ Mécanique } & Confinement & $\uparrow$ & Aorte & [28] \\
\hline & Rigidité & $\uparrow$ & Sinusoïdes hépatiques & [31] \\
\hline
\end{tabular}

Tableau I. Différents types de signaux régulent la formation des podosomes. De multiples facteurs sont capables de moduler la formation des podosomes notamment dans le cas des cellules où les structures sont inductibles, comme les cellules endothéliales. Les signaux sont souvent d'origine extracellulaire (cytokines, nature et propriétés de la matrice, etc.) mais peuvent aussi résulter de changements internes (métabolisme, microARN, etc.). Ces régulations sont dynamiques car les podosomes agissent à leur tour sur le microenvironnement.

sement, sur une matrice rigide, leur nombre augmente et leur pouvoir protéolytique est multiplié [23, 31].

\section{- Les signaux émis par la cellule}

La formation des podosomes est favorisée par le relâchement de la tension cellulaire [32, 33], qui pourrait être propice à leur fonction de mécanosenseurs. Certains micro-ARN modulent la formation des podosomes par leurs effets sur la transcription de molécules modulant leur induction [34, 35]. La signalisation Notch, qui oriente le destin cellulaire également par voie transcriptionnelle, est apparue comme une nouvelle voie de régulation de la formation des podosomes [36]. Des modifications chimiques (carence en di-oxygène ou en nutriments [37], ou production d'espèces réactives de l'oxygène [38]), qui affectent le métabolisme, peuvent déclencher l'assemblage des podosomes dont le rôle, dans ces situations, n'apparaît pas encore clairement (Tableau I). II est vraisemblable que leur assemblage ne procède pas dans ce cas de la même façon que lors d'une stimulation extracellulaire.

Les podosomes des macrophages dans la migration trans-tissulaire La majorité des macrophages tissulaires sont issus de monocytes sanguins qui se différencient après infiltration des tissus. Leur mis- sion: débarrasser l'organisme des pathogènes et des déchets. Ils sont ainsi confrontés à des matrices dont ils doivent décoder la composition, la rigidité et la topographie pour une migration «tout terrain ». Dans des tissus poreux, comme celui formé par un réseau lâche de fibres de collagène-I, leur déformabilité suffit à leur progression et leur mouvement est alors de type amiboïde. Lorsque le réseau de fibres est dense, les macrophages forment des podosomes. Ils utilisent alors les propriétés protéolytiques de ces podosomes pour se frayer un passage dans le stroma, selon un mode de migration de type mésenchymal [39]. Les monocytes peuvent se différencier en deux types de macrophages de phénotypes et de fonctions distincts : les macrophages $\mathrm{Ml}$, associés à l'exposition aux stimulus de la phase précoce des réponses inflammatoire et immunitaire, et les macrophages M2, impliqués dans les phases plus tardives de reconstitution. Bien que les deux populations présentent des podosomes, seuls ceux des macrophages M2, plus dynamiques que ceux des macrophages $\mathrm{Ml}$, participent à la mobilité mésenchymale [40]. 
In vitro, sur une surface bidimensionnelle, l'adhérence est une fonction proéminente des podosomes pour les macrophages qui sont dépourvus d'adhérence focales. Les podosomes apparaissent alors uniformément distribués à la face ventrale des cellules. En réponse à un facteur chémotactique (par exemple la chimiokine CSF1), les cellules deviennent mobiles et les podosomes qui se désassemblent et se reforment en continu s'accumulent au front de migration. La dynamique élevée des podosomes des macrophages M2 permet leur migration et la traversée de l'endothélium [8]. Les macrophages possèdent deux populations de podosomes : (1) les précurseurs, de grande taille et dynamiques, localisés en périphérie et au front de migration; et (2) les successeurs, plus petits, plus stables, en arrière des premiers, qui résultent souvent de leur scission [18]. Ces différences pourraient être inhérentes à la régulation différentielle de la myosine-Il par les composants de la coiffe : LSP1 pour les précurseurs, supervilline pour les successeurs [19].

Le cas particulier d'un endothélium ou de la lame basale peut être représenté par une situation en deux dimensions. Cependant, dans un gel de collagène-I, mimant l'environnement des macrophages dans les tissus in vivo, les podosomes ont une présentation bien différente. On les appellent alors podosomes 3D [41]. Ils se forment à l'extrémité de longues protrusions et sont identifiés par leur fonction protéolytique, leur dynamique et leurs marqueurs, car la structuration cœur/anneau/ chapeau n'est alors plus discernable. Ils sont également moins nombreux, plus massifs et de composition légèrement différente. In vitro, les macrophages porteurs de podosomes 3D et présentant la MTl-MMP peuvent créer des tunnels dans un gel de Matrigel ou de collagène-I (Figure 2) [42] $(\rightarrow$ ). $(\rightarrow)$ Voir la Synthèse de $V$. Le Cabec et al., $m / s n^{\circ} 12$, décembre 2011, page 1112
Ce rôle des podosomes dans l'invasion transtissulaire est en accord avec des observations réalisées in vivo. En effet, en manipulant la stabilité des podosomes de macrophages chez la souris, leur taille, mais aussi leur potentiel de dégradation sont altérés, et ce gain de fonction pourrait rendre compte de la migration accélérée des macrophages ainsi modifiés vers les sites d'inflammation in vivo [43-45]. Il est probable que les podosomes 3D aient également les propriétés d'adhérence décrites en deux dimensions: des intégrines et le récepteur de l'acide hyaluronique et du collagène-I CD44 sont en effet localisés au niveau de ces structures [41], mais la fonction de mécanosenseur et de transduction des signaux matriciels qu'ils pourraient y exercer est plus difficile à explorer.

\section{Les podosomes des cellules dendritiques : une fonction dans la présentation de l'antigène}

Les cellules dendritiques sont les principales cellules qui captent au niveau des tissus et du sang les éléments étrangers afin de les présenter, dans les ganglions, aux lymphocytes $T$ et éduquer ces derniers afin de déclencher une réponse immunitaire adaptée. Ce sont des cellules présentatrices d'antigènes professionnelles. Il existe plusieurs sous-populations : insterstitielles, dermiques, plasmocytoïdes, infammatoires. Les cellules de Langerhans, présentes dans l'épiderme, font partie de ce type de cellules. En l'absence de signal déclencheur (pathogènes, agresseur, etc.), ces cellules sont dites immatures (iDC) et au repos. Elles sondent constamment le microenvironnement à la recherche d'antigènes grâce

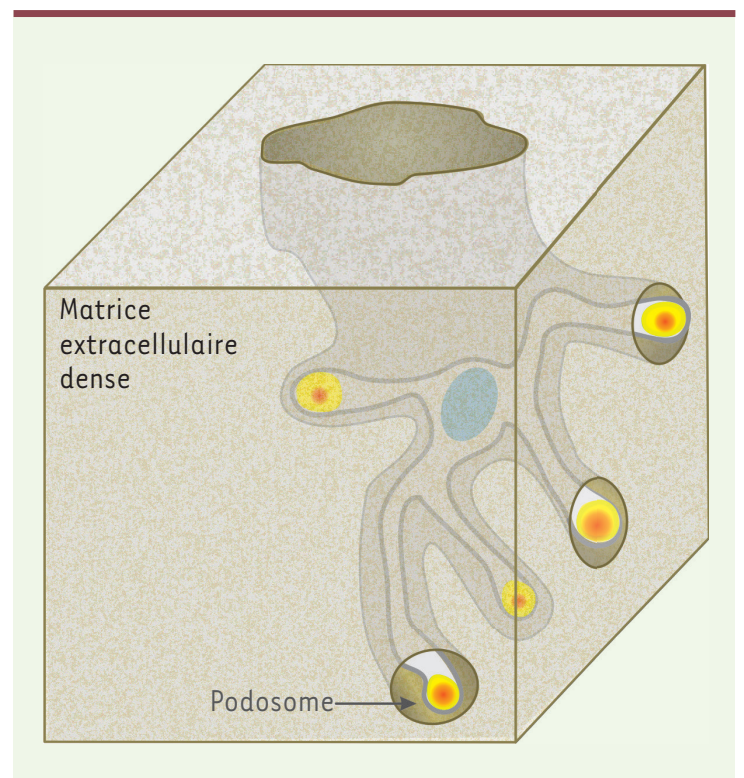

Figure 2. Les podosomes permettent aux macrophages de creuser des tunnels dans la matrice pour infiltrer les tissus. Dans une matrice tridimensionnelle et compacte de collagène-I (montrée sous forme de bloc), les macrophages forment des protrusions avec des podosomes de forme globulaire à leurs extrémités. Ces podosomes dits 3D ne présentent pas l'organisation cœur/anneau qui caractérise les podosomes dans une situation 2D (voir Figure 1A). La protéolyse matricielle des podosomes, associée aux mouvements des protrusions, conduit à la formation d'un tunnel. S'ils se vérifient in vivo, ces évènements pourraient permettre la migration transtissulaire des macrophages eux-mêmes mais également d'autres cellules.

aux PRR (pattern recognition receptors) qu'elles expriment et qui reconnaissent des motifs associés en particulier aux pathogènes bactériens, fongiques ou viraux (PAMP, pour pathogen-associated molecular patterns) [46]. L'interaction entre PRR et PAMP déclenche alors l'internalisation de l'antigène par endocytose, ou la phagocytose du pathogène, conduisant à une réponse immunitaire innée (caractérisée en particulier par une production de cytokines) et la présentation des antigènes aux lymphocytes via les protéines du complexe majeur d'histocompatibilité de type II.

In vitro, les iDC présentent des regroupements de podosomes qui sont disposés en bouquets (Figure 1A). Durant la phase de maturation qui suit l'interaction avec les antigènes, les podosomes disparaissent au bénéfice de la formation de longs dendrites (d'où le nom de ces cellules). Cette transformation, qui réduit l'adhérence des cellules à la matrice extracellulaire, marque la transition vers un mode de migration rapide des cellules pour gagner les ganglions lymphatiques, siège de l'activation des lymphocytes T [47]. 


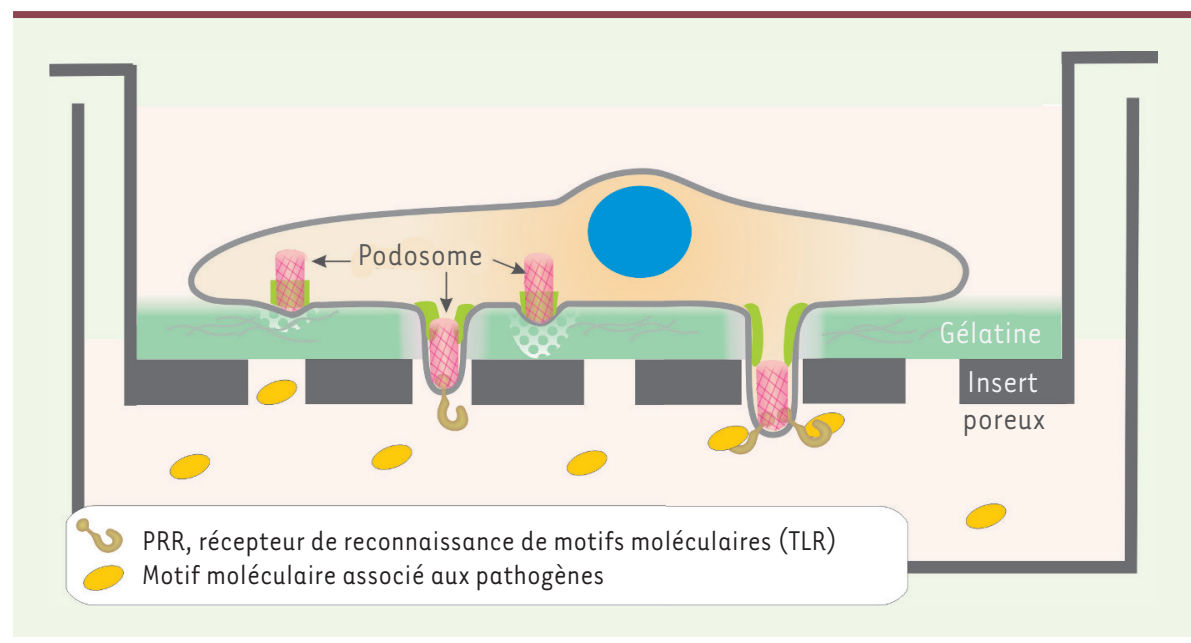

Figure 3. Les podosomes des cellules dendritiques immatures participent à la reconnaissance et au traitement des antigènes. Lorsque les cellules sont ensemencées sur le filtre de polycarbonate recouvert de gélatine (dans une chambre de Boyden), les podosomes se forment au-dessus des pores car les modifications de topographie amorcent la construction du podosome et déterminent sa morphologie: l'anneau de protéines adhésives reste positionné au niveau du filtre mais le cœur de la structure s'engage dans le pore. Cette extension du cœur d'actine est dépendante de l'activité de la myosine-II. La protrusion qui maintenant expose la métalloprotéinase matricielle MTl-MMP et les PRR (pattern recognition receptor) devient un site privilégié de protéolyse matricielle, de capture d'antigènes et d'endocytose. Si elles se vérifient in vivo, cette architecture et cette fonction des podosomes pourraient permettre aux cellules dendritiques immatures (iDC) de dénicher des pathogènes à distance, par exemple au contact des épithéliums.

Les podosomes participent spécifiquement à la phase de reconnaissance et de traitement de l'antigène par les iDC. Sur une membrane de polycarbonate, les podosomes des iDC se forment au-dessus des pores de la membrane $[30,48]$. Les modifications de la topographie rencontrées par la cellule semblent agir comme un signal initiant la construction du podosome, peut-être en recrutant des protéines de détection de courbure dans la membrane plasmique [49]. La topographie impacte aussi la morphologie du podosome: l'anneau de protéines adhésives (vinculine, paxilline, taline et autres composants) reste positionné au niveau du filtre mais le cœur de la structure, lui, s'engage dans le pore (Figure 3 ). Cette extension du cœur d'actine dépend de l'activité de la myosine-II. Cette protrusion expose alors MTl-MMP et les PRR : c'est ainsi un site privilégié de protéolyse matricielle, de capture des antigènes et d'endocytose $[30,48]$. Cette architecture et cette fonction des podosomes pourraient, si elles se vérifient in vivo, permettre aux iDC de dénicher des pathogènes à distance, par exemple au contact de l'épithélium intestinal ou pulmonaire [48].

\section{Les podosomes des cellules endothéliales et l'angiogenèse}

Les cellules endothéliales tapissent la face interne de tous les vaisseaux sanguins. Elles jouent un rôle essentiel dans l'angiogenèse, un processus qui conduit à l'extension du réseau vasculaire. Celui-ci implique principalement les micro-vaisseaux qui bourgeonnent pour instaurer une vascularisation lors du développement, ou restaurer l'irrigation de tissus lésés ou ischémiques. Le processus et ses différentes étapes sont bien caractérisés car celles-ci peuvent être étudiées en détail ex vivo dans le modèle de la rétine du souriceau nouveau-né, chez lequel la vascularisation débute au cours de la première semaine de vie. Les cellules endothéliales réagissent au facteur angiogénique VEGF. Celles qui expriment fortement le récepteur de ce facteur acquièrent un phénotype particulier (ou tip cells).
Elles prennent en effet le rôle de leader en imposant aux cellules adjacentes un phénotype distinct (les stalk cells), sous le contrôle de la voie Notch, activée par l'expression du ligand membranaire de Notch DII4 (delta-like 4). Les cellules tip présentent des filopodes qui explorent l'environnement et, garnies de récepteurs pour capter le VEGF et d'intégrines pour s'agripper à la matrice extracellulaire, elles commencent leur migration. Les cellules voisines, les stalk cells, ne migrent pas mais prolifèrent. Ces différences phénotypiques et comportementales sont associées à des profils d'expression génique distincts orchestrés par la signalisation Notch. $\varepsilon n$ culture, les cellules endothéliales microvasculaires forment des podosomes lorsqu'elles sont exposées au VEGF [26, 36, 50-52]. Ces podosomes sont interconnectés par des câbles d'acto-myosine dans une superstructure de forme annulaire souvent appelée rosette [36]. Ils ont une forte activité protéolytique, notamment sur une matrice de collagène-IV [26]. L'inhibition de la voie Notch induit également podosomes et protéolyse matricielle [36].

Le modèle de la rétine du souriceau nouveau-né a été utilisé pour comprendre l'implication des podosomes dans la biologie vasculaire et les expériences d'immunofluorescence, réalisées avec des marqueurs de podosomes, ont permis de les révéler dans les cellules endothéliales tip. Dans leur microenvironnement natif, au contact du collagène-IV et de la laminine de la lame basale, les podosomes sont des structures globulaires [36]. L'analyse de ces composants matriciels révèle l'existence d'une protéolyse sélective du collagène-IV. 


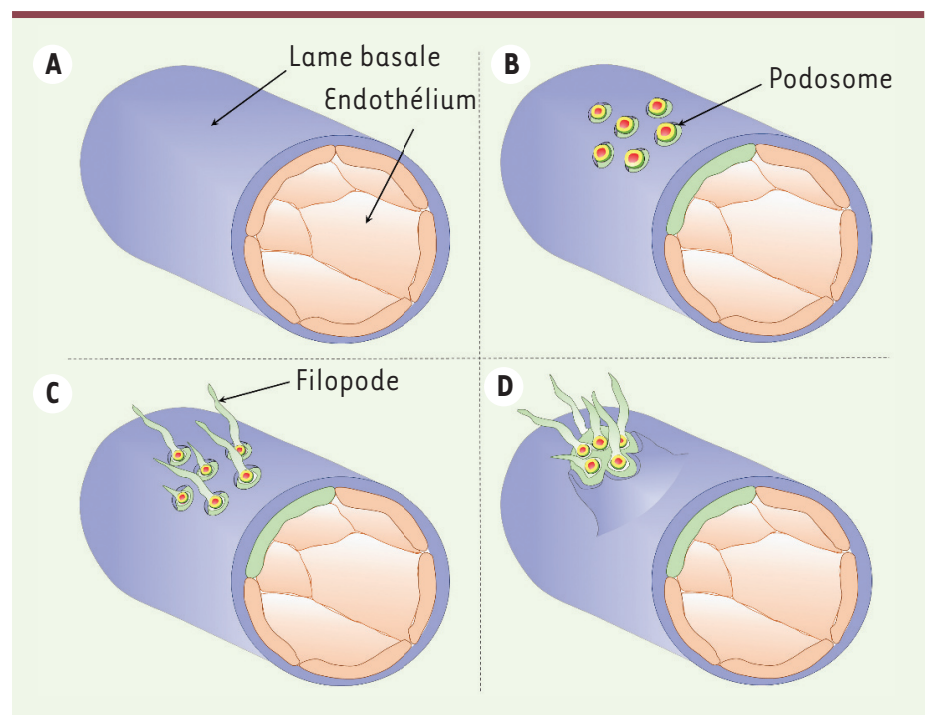

Figure 4. Les podosomes des cellules endothéliales digèrent localement la lame basale lors de l'angiogenèse. Les cellules endothéliales acquièrent des propriétés invasives pour permettre l'émergence de ramifications des vaisseaux sanguins. $\boldsymbol{A}$. Les cellules endothéliales reposent sur la lame basale; en absence de stimulus pro-angiogénique, celle-ci leur apporte des signaux de survie et de quiescence. B. En réponse au VEGF (vascular endothelial growth factor), certaines cellules se transforment (tip cell, cellule verte) et assemblent des podosomes qui dégradent localement la lame basale sous-jacente. C. Des filopodes émergent au niveau des brèches ainsi créées. $D$. La cellule bourgeonne et les filopodes agrippent la matrice extracellulaire par l'engagement d'intégrines, permettant à cette cellule de s'extraire de son vaisseau d'origine pour donner naissance à une ramification vasculaire.

Les podosomes pourraient ainsi être à l'origine de l'ouverture d'une brèche dans la lame basale et permettre l'extrusion des cellules tip et l'émergence d'une ramification du réseau de capillaires (Figure 4) mais également décaper la matrice à la surface d'un vaisseau à proximité pour favoriser l'anastomose de deux capillaires.

Les câbles interconnectant les podosomes sont clairement visibles dans les cellules tip, suggérant un comportement coordonné permettant aux cellules de franchir la barrière que constitue la lame basale. $\varepsilon n$ arrière de ces cellules, la voie Notch est active et la formation des podosomes est réprimée [36].

\section{Conclusions et perspectives}

Notre connaissance des podosomes a considérablement progressé aux cours des dernières années, notamment grâce aux développements technologiques de la microscopie [53]. La mise en évidence des structures in vivo lors de l'angiogenèse fait des podosomes des cibles thérapeutiques potentielles dans l'angiogenèse pathologique. L'intervention des podosomes dans les autres processus décrits ici reste à démontrer in vivo, mais pourrait permettre de limiter l'infiltration tissulaire des macrophages et, en conséquence, l'inflammation dans les maladies chroniques et les cancers, ou encore de contrôler certains aspects de l'immunité. Les processus impliquant les podosomes ne se limitent pas aux situations présentées. La formation de la zone de scellement dans les ostéoclastes engagés dans la résorption osseuse [4], la maturation de la plaque post-synaptique dans le système neuromusculaire [54] ou la fusion des myoblastes [55] sont d'autres exemples des fonctions établies ou présumées de ces structures.

Les conditions d'observation des podosomes in vitro ont révélé leurs multiples potentialités, mais il est probable que les différentes fonctions des podosomes ne s'exercent pas simultanément. Les composantes du microenvironnement régulent l'assemblage, la morphologie et la composition des podosomes. Il est donc attendu qu'elles déterminent également leur(s) fonction(s). $\diamond$

\section{SUMMARY}

Variations on the theme of podosomes, context matters

Podosomes are actin-based microdomains connecting the cell with its extracellular matrix. Contractile actinmyosin cables assemble them into a network that constitutes a versatile cellular superstructure. Discovered and extensively described in in vitro conditions, podosomes now appear as major actors of specific physiological processes. They share common characteristics but their morphology and their effect on cell functioning can only be apprehended in specific cellular contexts. We focus here on three cellular processes involving podosomes and discuss their properties in context. $\diamond$

\section{REMERCIEMENTS}

Les auteurs s'excusent auprès des nombreux scientifiques dont le travail n'a pas pu être correctement discuté et référencé pour respecter le format. Le laboratoire (http://genot-lab.org/) est financé par la Ligue contre le Cancer, comité de la Gironde, l'Université de Bordeaux (projet transversal HYPOXCELL), l'agence nationale de la Recherche ANR-17-CE11-0033-01 et l'association Marfans. FA est financé par la fondation Berthe Fouassier-maladies de l'œil, sous l'égide de la Fondation de France.

Le Consortium Invadosome est un réseau international unissant des laboratoires s'intéressant aux structures d'adhérence impliquées dans les processus invasifs. Il est ouvert à l'ensemble de la communauté scientifique (http://www.invadosomes.org/).

\section{LIENS D'INTÉRÊT}

Les auteurs déclarent n'avoir aucun lien d'intérêt concernant les données publiées dans cet article.

\section{RÉFÉRENCES}

1. Veillat V, Spuul P, Daubon T, et al. Podosomes: multipurpose organelles? Int J Biochem Cell Biol $2015 ; 65: 52-60$.

2. Rowe RG, Weiss SJ. Breaching the basement membrane: who, when and how? Trends Cell Biol $2008 ; 18: 560-74$. 


\section{RÉFÉRENCES}

3. Labernadie A, Bouissou A, Delobelle P, et al. Protrusion force microscopy reveals oscillatory force generation and mechanosensing activity of human macrophage podosomes. Nat Commun 2014 $5: 5343$.

4. Luxenburg C, Geblinger D, Klein $\varepsilon$, et al. The architecture of the adhesive apparatus of cultured osteoclasts: from podosome formation to sealing zone assembly. PLoS ONE 2007 ; 2 : el79.

5. van den Dries K, Meddens MB, de Keijzer S, et al. Interplay between myosin IIA-mediated contractility and actin network integrity orchestrates podosome composition and oscillations. Nat Commun $2013 ; 4$ : 1412.

6. Proag A, Bouissou A, Mangeat T, et al. Working together: spatial synchrony in the force and actin dynamics of podosome first neighbors. ACS Nano $2015 ; 9$ : 3800-13.

7. Linder S, Nelson D, Weiss M, Aepfelbacher M. Wiskott-Aldrich syndrome protein regulates podosomes in primary human macrophages. Proc Natl Acad Sci U S A $1999 ; 96$ : 9648-53.

8. Burns $S$, Thrasher AJ, Blundell MP, et al. Configuration of human dendritic cell cytoskeleton by Rho GTPases, the WAS protein, and differentiation. Blood 2001 ; 98 : 1142-9.

9. Destaing 0, Saltel F, Geminard JC, et al. Podosomes display actin turnover and dynamic selforganization in osteoclasts expressing actin-green fluorescent protein. Mol Biol Cell 2003; 14 407-16.

10. Schachtner H, Calaminus SD, Sinclair A, et al. Megakaryocytes assemble podosomes that degrade matrix and protrude through basement membrane. Blood $2013 ; 121: 2542-52$.

11. Wiesner C, Faix J, Himmel M, et al. KIF5B and KIF3A/KIF3B kinesins drive MT1-MMP surface exposure, $\mathrm{CD} 44$ shedding, and extracellular matrix degradation in primary macrophages. Blood $2010 ; 116: 1559-69$.

12. عl Azzouzi K, Wiesner C, Linder S. Metalloproteinase MTl-MMP islets act as memory devices for podosome reemergence. J Cell Biol $2016 ; 213: 109-25$.

13. Saltel F, Daubon T, Juin A, et al. Invadosomes: intriguing structures with promise. Eur J Cell Biol $2011 ; 90: 100-7$

14. Walde M, Monypenny J, Heintzmann R, et al. Vinculin binding angle in podosomes revealed by high resolution microscopy. PLoS One 2014 ; 9 : e88251.

15. Cox S, Rosten $\varepsilon$, Monypenny J, et al. Bayesian localization microscopy reveals nanoscale podosome dynamics. Nat Methods 2011 ; 9 : 195-200.

16. Mersich AT, Miller MR, Chkourko H, Blystone SD. The formin FRLI (FMNLI) is an essential component of macrophage podosomes. Cytoskeleton (Hoboken) $2010 ; 67: 573-85$.

17. Panzer L, Trube L, Klose M, et al. The formins FHODI and INF2 regulate inter- and intra-structural contractility of podosomes. J Cell Sci $2016 ; 129: 298-313$

18. Bhuwania R, Cornfine S, Fang Z, et al. Supervillin couples myosin-dependent contractility to podosomes and enables their turnover. J Cell Sci 2012; 125:2300-14.

19. Cervero P, Wiesner C, Bouissou A, et al. Lymphocyte-specific protein 1 regulates mechanosensory oscillation of podosomes and actin isoform-based actomyosin symmetry breaking. Nat Commun $2018 ; 9: 515$.

20. Linder S, Higgs $H$, Hufner $K$, et al. The polarization defect of Wiskott-Aldrich syndrome macrophages is linked to dislocalization of the Arp2/3 complex. J Immunol $2000 ; 165: 221-5$.

21. Akisaka T, Yoshida H, Suzuki R, Takama K. Adhesion structures and their cytoskeleton-membrane interactions at podosomes of osteoclasts in culture. Cell Tissue Res 2008 ; $331: 625-41$.

22. Labernadie A, Thibault C, Vieu C, et al. Dynamics of podosome stiffness revealed by atomic force microscopy. Proc Natl Acad Sci U S A 2010 ; 107 : 21016-21.

23. Bouissou A, Proag A, Bourg N, et al. Podosome force generation machinery: a local balance between protrusion at the core and traction at the ring. ACS Nano 2017 ; 11 : 4028-40.

24. Burgstaller $G$, Gimona M. Podosome-mediated matrix resorption and cell motility in vascular smooth muscle cells. Am J Physiol Heart Circ Physiol 2005 ; 288 : H3001-5

25. Horton MA, Nesbit MA, Helfrich MH. Interaction of osteopontin with osteoclast integrins. Ann N Y Acad Sci $1995 ; 760: 190-200$

26. Daubon T, Spuul P, Alonso F, et al. VEGF-A stimulates podosome-mediated collagen-IV proteolysis in microvascular endothelial cells. J Cell Sci $2016 ; 129: 2586-98$.

27. Juin A, Billottet C, Moreau V, et al. Physiological type I collagen organization induces the formation of a novel class of linear invadosomes. Mol Biol Cell $2012 ; 23: 297-309$.

28. Spuul P, Chi Py, Billottet $C$, et al. Microfluidic devices for the study of actin cytoskeleton in constricted environments: Evidence for podosome formation in endothelial cells exposed to a confined environment. Methods $2016 ; 94: 65-74$.

29. van den Dries K, van Helden SF, te Riet J, et al. Geometry sensing by dendritic cells dictates spatial organization and PGE(2)-induced dissolution of podosomes. Cell Mol Life Sci $2012 ; 69$ : 1889-901.

30. Gawden-Bone C, Zhou Z, King $\varepsilon$, et al. Dendritic cell podosomes are protrusive and invade the extracellular matrix using metalloproteinase MMP-14. J Cell Sci $2010 ; 123: 1427-37$.

31. Juin $A$, Planus $\varepsilon$, Guillemot $F$, et al. Extracellular matrix rigidity controls podosome induction in microvascular endothelial cells. Biol Cell $2013 ; 105: 46-57$.

32. Moreau V, Tatin F, Varon C, Genot $\varepsilon$. Actin can reorganize into podosomes in aortic endothelial cells, a process controlled by Cdc42 and RhoA. Mol Cell Biol $2003 ; 23: 6809-22$.

33. $\mathrm{Yu} \mathrm{CH}_{\mathrm{C}}$, Rafiq NB, Krishnasamy A, et al. Integrin-matrix clusters form podosome-like adhesions in the absence of traction forces. Cell Rep $2013 ; 5: 1456-68$.
34. Curado F, Spuul P, Egana I, et al. ALK5 and ALKl play antagonistic roles in transforming growth factor beta-induced podosome formation in aortic endothelial cells. Mol Cell Biol $2014 ; 34: 4389-403$.

35. Quintavalle M, Elia L, Condorelli G, Courtneidge SA. MicroRNA control of podosome formation in vascular smooth muscle cells in vivo and in vitro. $J$ Cell Biol $2010 ; 189$ : 13-22.

36. Spuul P, Daubon T, Pitter B, et al. VEGF-A/Notch-induced podosomes proteolyse basement membrane collagen-IV during retinal sprouting angiogenesis. Cell Rep 2016; $17: 484-500$.

37. VanWinkle WB, Snuggs M, Buja LM. Hypoxia-induced alterations in cytoskeleton coincide with collagenase expression in cultured neonatal rat cardiomyocytes. J Mol Cell Cardiol $1995 ; 27: 2531-42$

38. Mu X, Wang X, Huang W, et al. Circulating exosomes isolated from septic mice induce cardiovascular hyperpermeability through promoting podosome cluster formation. Shock $2018 ; 49: 429-41$.

39. Van Goethem $\varepsilon$, Poincloux R, Gauffre F, et al. Matrix architecture dictates three-dimensional migration modes of human macrophages: differential involvement of proteases and podosome-like structures. J Immunol 2010; $184: 1049-61$.

40. Cougoule C, Van Goethem E, Le Cabec V, et al. Blood leukocytes and macrophages of various phenotypes have distinct abilities to form podosomes and to migrate in 3D environments. Eur J Cell Biol 2012; 91 : 938-49.

41. Van Goethem $\varepsilon$, Guiet R, Balor S, et al. Macrophage podosomes go 3D. Eur J Cell Biol $2011 ; 90: 224-36$.

42. Le Cabec V, Van Goethem $\varepsilon$, Guiet R, Maridonneau-Parini I. La migration des phagocytes : tour d'horizon. Med Sci (Paris) 2011 ; 27 : 1112-9.

43. Cougoule C, Le Cabec V, Poincloux R, et al. Three-dimensional migration of macrophages requires Hck for podosome organization and extracellular matrix proteolysis. Blood 2009; 115 : 1444-52.

44. Verollet C, Le Cabec V, Maridonneau-Parini I. HIV-1 Infection of T Lymphocytes and Macrophages Affects Their Migration via Nef. Front Immunol $2015 ; 6: 514$

45. Verollet $C$, Souriant $S$, Bonnaud $\varepsilon$, et al. HIV-1 reprograms the migration of macrophages. Blood $2015 ; 125: 1611-22$

46. Lipscomb MF, Masten BJ. Dendritic cells: immune regulators in health and disease. Physiol Rev $2002 ; 82$ : 97-130.

47. van Helden SF, Krooshoop DJ, Broers KC, et al. A critical role for prostaglandin $\varepsilon 2$ in podosome dissolution and induction of high-speed migration during dendritic cell maturation. J Immunol 2006 ; 177 : 1567-74.

48. Baranov MV, Ter Beest M, Reinieren-Beeren I, et al. Podosomes of dendritic cells facilitate antigen sampling. J Cell Sci 2014 ; 127 : 1052-64.

49. Gallop JL, McMahon HT. BAR domains and membrane curvature: bringing your curves to the BAR. Biochem Soc Symp 2005 : 223-31.

50. Fey T, Schubert KM, Schneider H, et al. Impaired endothelial shear stress induces podosome assembly via VEGF up-regulation. FASEB J 2016 ; 30 : 2755-66.

51. Osiak AE, Zenner G, Linder S. Subconfluent endothelial cells form podosomes downstream of cytokine and RhoGTPase signaling. Exp Cell Res 2005 ; 307 : $342-53$.

52. Wang J, Taba Y, Pang J, et al. GITl mediates VEGF-induced podosome formation in endothelial cells: critical role for PLCgamma. Arterioscler Thromb Vasc Biol $2009 ; 29$ : 202-8.

53. Meddens MB, van den Dries K, Cambi A. Podosomes revealed by advanced bioimaging: what did we learn? Eur J Cell Biol 2014 ; 93 : 380-7.

54. Proszynski TJ, Gingras J, Valdez G, et al. Podosomes are present in a postsynaptic apparatus and participate in its maturation. Proc Natl Acad Sci U S A 2009; 106 : 18373-8.

55. Chen $\varepsilon H$. Invasive podosomes and myoblast fusion. Curr Top Membr 2011 ; $68: 235-58$.

56. Varon C, Tatin F, Moreau V, et al. Transforming growth factor beta induces rosettes of podosomes in primary aortic endothelial cells. Mol Cell Biol $2006 ; 26: 3582-94$
TIRÉS À PART

દ. Génot 